\title{
Lung inflammation and immunity: report from the 12th ERS Lung Science Conference
}

\author{
Bruno Crestani $i^{1,2,3}$, Marina Pretolani $i^{1,2,3}$ and Hamida Hammad ${ }^{4}$
}

Affiliations: ${ }^{1}$ Inserm, U1152, LabEx Inflamex, Paris, France. ${ }^{2}$ Université Paris Diderot, Sorbonne Paris Cité, Paris, France. ${ }^{3}$ Service de Pneumologie, APHP, Hôpital Bichat, DHU Fire, Paris, France. ${ }^{4}$ Laboratory of Immunoregulation and Mucosal Immunology, Inflammation Research Center-VIB, Ghent University, Ghent, Belgium.

Correspondence: Bruno Crestani, Service de Pneumologie A, Hôpital Bichat, 46 rue Henri Huchard, 75018 Paris, France. E-mail: bruno.crestaniabch.aphp.fr

-

@ERSpublications

The Lung Science Conference, organised by the ERS, showcases cutting edge respiratory research http://ow.ly/xN29D

The European Respiratory Society (ERS) seeks to alleviate suffering from respiratory diseases and promote lung health through research, dissemination of knowledge, and medical and public education. In support of this mission, the ERS organises the annual Lung Science Conference (LSC). This world-class research meeting brings together leading experts on topics that are of relevance to respiratory medicine. Furthermore, at the LSC, one of the main focuses is early-career physicians and scientists who are involved in respiratory research, as these are the future leaders of the field [1].

Inflammatory diseases of the lung are a major cause of morbidity and mortality. In March 2014, international experts met for the 12th LSC in Estoril, Portugal, to present and discuss novel findings in the field of "Lung Inflammation and Immunity", recognising that inflammation is the main pathogenic process in many respiratory diseases, such as asthma and infection, and a significant contributor to many others $[2,3]$. Old and recent anti-inflammatory and/or immunomodulating drugs are the main tools of our current treatment in many respiratory diseases, and new drugs targeting inflammatory pathways are being developed. This was discussed in an early evening session "The new drug discovery paradigm" by Tomas Mustelin (Respiratory, Inflammatory, and Autoimmune Diseases, MedImmune, Gaithersburg, MD, USA) and Robert Strieter (Novartis Institutes for BioMedical Research, Cambridge, MA, USA), who were both involved in academic respiratory research before joining pharmaceutical companies. Part of the 2014 LSC was focused on the mechanisms of allergy and asthma, but other fields of interest were discussed, particularly lung fibrosis, chronic obstructive pulmonary disease and emphysema, pulmonary hypertension, and lung transplantation.

Initiation and maintenance of the allergic response in the respiratory tract

Inflammation and immunity are central to the pathogenesis of asthma and other allergic diseases. As discussed by Bart Lambrecht (Ghent University, Ghent, Belgium), dendritic cells drive the immune process very early in the disease through their capacity to steer the immune response. In response to allergen presentation by airway dendritic cells, T-helper (Th) lymphocytes of the adaptive immune system control many aspects of asthma development via secretion of interleukin (IL)-4, IL-5, IL-13, IL-17 and IL-22, and these are counterbalanced by cytokines produced by regulatory T-cells. Remy Bosselut (National Institutes of Health, Bethesda, MD, USA) showed that T-cell differentiation is a complex issue with transcriptional

Received: June 052014 | Accepted: June 052014

Conflict of interest: None declared.

Provenance: Submitted article, peer reviewed.

Copyright OERS 2014. ERR articles are open access and distributed under the terms of the Creative Commons Attribution Non-Commercial Licence 4.0. 
circuits directing helper versus cytotoxic programmes [4]. Many cells of the innate immune system, such as mast cells, basophils, neutrophils, eosinophils and innate lymphoid cells, also play an important role in disease pathogenesis. Barrier epithelial cells are more important in disease pathogenesis than previously thought. In the past years, these cells have been shown to sense allergens via pattern recognition receptors, protease-activated receptors and lectin receptors. They can also activate conventional and inflammatorytype dendritic cells and other innate immune cells through the secretion of thymic stromal lymphopoietin, granulocyte-macrophage colony-stimulating factor, IL-1, IL-33 and IL-25. Understanding this cytokine crosstalk between barrier epithelial cells, dendritic cells and immune cells provides important insights into the mechanisms of allergic sensitisation and asthma progression [5]. In the skin, the crosstalk between epithelial cells (keratinocytes) and immune cells may contribute to the "atopic march" observed in patients, with a crucial role for keratinocytic thymic stromal lymphopoietin, by promoting allergen sensitisation occurring in barrier-impaired skin, which ultimately leads to allergic asthma [6]. This is discussed in this issue of the European Respiratory Review by Li [7]. Similarly, alterations in the way intestinal epithelial cells sense gut bacteria can lead to severe inflammation in the gastrointestinal tract as evidenced by Manolis Pasparakis (University of Cologne, Cologne, Germany) $[8,9]$.

The role of mast cells and basophils in allergic and inflammatory diseases was discussed by Jonas Erjefält (Lund University of Lund, Lund, Sweden) and Hajime Karasuyama (Tokyo Medical and Dental University, Tokyo, Japan) respectively. Both presented very new data on these relatively underestimated cells. Jonas Erjefalt showed that specific populations of mast cells expressing high levels of IgE receptors were present in the alveolar parenchyma of patients with allergic asthma only. This is discussed further in this issue of the European Respiratory Review [10]. This population has never been observed in patients with allergic rhinitis or chronic obstructive pulmonary disease. Hajime Karasuyama reported that basophils can control monocyte differentiation into cells with anti-inflammatory properties. This was explained by the fact that basophils can express CCR2, a chemokine receptor normally expressed by monocytes, which can therefore be recruited very quickly to the site of inflammation. Basophils, through their IL-4 production, can favour a M2 phenotype in differentiating monocytes. Pierre Bruhns (Institute Pasteur, Paris, France) discussed the key roles for IgG, IgG receptors and neutrophils in anaphylaxis in mice and humans $[11,12]$.

As discussed by Andrew McKenzie (Medical Research Council Laboratory of Molecular Biology, Cambridge, UK), until recently, the primary contributors of type 2 cytokines were believed to be Th2 cells. This concept was challenged by the discovery of group 2 innate lymphoid cells (ILC2s) in the lung, which represent a major source of type 2 cytokines during the acute inflammatory phase. Recent advances in our understanding of the regulation and development of ILC2s have redrawn the roadmap of type 2 inflammation. Indeed, ILC2-derived IL-13 production appears to be critical for the induction of Th2 immunity, mainly by controlling dendritic cell migration to the T-cell area of the draining lymph nodes. Thus, ILC2 plays a central role in immune regulation. Because ILC2s act upstream of the entire Th2 cascade, they might serve as the early tile in a Th2 domino effect. As such, ILC2s represent a novel attractive target for future drug development [13].

\section{Inflammation and immunity besides allergy and asthma}

Inflammation and immunity participate in the pathophysiology of pulmonary arterial hypertension (PAH) $[14,15]$, as discussed by Frederic Perros (Université Paris-Sud, Le Plessis Robinson, France). Inflammation is involved in the initiation and the maintenance of vascular remodelling in most of the animal models of $\mathrm{PAH}$, and therapeutic targeting of inflammation in these models blocks PAH development. In humans, pulmonary vascular lesions of PAH are the source of cytokine and chemokine production related to inflammatory cell recruitment and lymphoid neogenesis. Circulating autoantibodies to endothelial cells and fibroblasts have been reported in $10-40 \%$ of patients with idiopathic $\mathrm{PAH}$, suggesting a possible role for autoimmunity in the pathogenesis of pulmonary vascular lesions. Current specific PAH treatments have immunomodulatory properties, and some studies have demonstrated a correlation between levels of circulating inflammatory mediators and patient survival.

Besides neutrophils and macrophages, the development of emphysema in humans and mice exposed to cigarette smoke is promoted by the activation of an adaptive immune response. Lung myeloid dendritic cells derived from cigarette smokers activate autoreactive Th1 and Th17 cells. Animal models have confirmed a significant role for autoimmunity in progressive smoke-induced emphysema. A proinflammatory pathway, in which reduced peroxisome proliferator activated receptor- $\gamma$ activity promotes emphysema, was highlighted by Farrah Kheradmand (Center for Translational Research on Inflammatory Diseases, Houston, TX, USA). In mice, targeting this pathway with a peroxisome proliferator activated receptor- $\gamma$ agonist reversed emphysema despite continual smoke exposure. These data presented by Farrah Kheradmand suggest that targeting this pathway in smokers could prevent and reverse emphysema [16, 17]. 
Lung transplantation is without doubt an immune condition wherein survival of the patient and the function of the transplanted organ depend on the daily usage of immunosuppressive drugs. The survival rate for lung transplantation is among the worst. Better understanding of T-cell regulation in lung transplantation may help us develop new therapeutic strategies in lung transplantation, as discussed by Stephen Juvet (John Radcliffe Hospital, Oxford, UK) [18].

\section{Resolving inflammation: the new frontier?}

Inflammatory responses, like all biological cascades, are shaped by a delicate balance between positive and negative feedback loops. It is now clear that the inflammatory cascade boasts a family of mediators that actively promote resolution and tissue repair without compromising host defence, as demonstrated by Derek Gilroy (University College London, London, UK). Indeed, the resolution phase of inflammation is actively orchestrated and carefully regulated, as are its induction and inhibition. The arachidonic acidderived prostaglandins, lipoxins and their natural C15-epimers are the main players in this area. In the future, treatment of inflammation might be not restricted to the use of inhibitors of the acute cascade but may take into account the therapeutic potential of inducers of the resolution phase of inflammation [19].

\section{Conclusion}

Inflammation is still at the heart of innovation in respiratory medicine. We need imaginative investigators to bring forward new concepts. The development of new imaging tools that allow, for example, the intravital imaging of the lung, as developed by Matthew F. Krummel (University of California, San Francisco, CA, USA) [20], may prove helpful, as the old saying goes that "seeing is believing". Our final aim remains to improve the respiratory health of our fellow human beings, and transfer from the research lab to the clinic is mandatory to achieve this task. The LSC is the place to be in order to reach this point. Join us in Estoril in March 2015 for the next instalment of the LSC, entitled "Lung Infection and Immunity".

\section{References}

1 Konigshoff M, Saglani S, Marsland BJ, et al. Rebuilding a diseased lung: repair and regeneration. Eur Respir J 2013; 41: 497-499.

2 Letuve S, Kozhich A, Humbles A, et al. Lung chitinolytic activity and chitotriosidase are elevated in chronic obstructive pulmonary disease and contribute to lung inflammation. Am J Pathol 2010; 176: 638-649.

3 Borensztajn K, Crestani B, Kolb M. Idiopathic pulmonary fibrosis: from epithelial injury to biomarkers - insights from the bench side. Respiration 2013; 86: 441-452.

4 Carpenter AC, Grainger JR, Xiong Y, et al. The transcription factors Thpok and LRF are necessary and partly redundant for $\mathrm{T}$ helper cell differentiation. Immunity 2012; 37: 622-633.

5 Lambrecht BN, Hammad H. Asthma: the importance of dysregulated barrier immunity. Eur J Immunol 2013; 43: 3125-3137.

6 Leyva-Castillo JM, Hener P, Jiang H, et al. TSLP produced by keratinocytes promotes allergen sensitization through skin and thereby triggers atopic march in mice. J Invest Dermatol 2013; 133: 154-163.

7 Li M. Current evidence of epidermal barrier dysfunction and thymic stromal lymphopoietin in the atopic march. Eur Respir Rev 2014; 23: 292-298.

8 Pasparakis M. Role of NF-kappaB in epithelial biology. Immunol Rev 2012; 246: 346-358.

9 Pasparakis M, Haase I, Nestle FO. Mechanisms regulating skin immunity and inflammation. Nat Rev Immunol 2014; 14: 289-301.

10 Erjefält JS. Mast cells in human airways: the culprit? Eur Respir Rev 2014; 23: 299-307.

11 Jonsson F, Mancardi DA, Kita Y, et al. Mouse and human neutrophils induce anaphylaxis. J Clin Invest 2011; 121: $1484-1496$.

12 Guilliams M, Bruhns P, Saeys Y, et al. The function of Fc $\gamma$ receptors in dendritic cells and macrophages. Nat Rev Immunol 2014; 14: 94-108.

13 Halim TY, McKenzie AN. New kids on the block: group 2 innate lymphoid cells and type 2 inflammation in the lung. Chest 2013; 144: 1681-1686.

14 Cohen-Kaminsky S, Hautefort A, Price L, et al. Inflammation in pulmonary hypertension: what we know and what we could logically and safely target first. Drug Discov Today 2014 [In press DOI: 10.1016/j.drudis.2014.04.007].

15 Hassoun PM. Inflammation in pulmonary arterial hypertension: is it time to quell the fire? Eur Respir J 2014; 43: 685-688.

16 Shan M, You R, Yuan X, et al. Agonistic induction of PPAR $\gamma$ reverses cigarette smoke-induced emphysema. J Clin Invest 2014; 124: 1371-1381.

17 Lea S, Plumb J, Metcalfe $\mathrm{H}$, et al. The effect of peroxisome proliferator-activated receptor- $\gamma$ ligands on in vitro and in vivo models of COPD. Eur Respir J 2014; 43: 409-420.

18 Juvet SC, Zhang L. Double negative regulatory $\mathrm{T}$ cells in transplantation and autoimmunity: recent progress and future directions. J Mol Cell Biol 2012; 4: 48-58.

19 Buckley CD, Gilroy DW, Serhan CN. Proresolving lipid mediators and mechanisms in the resolution of acute inflammation. Immunity 2014; 40: 315-327.

20 Gerard A, Khan O, Beemiller P, et al. Secondary T cell-T cell synaptic interactions drive the differentiation of protective $\mathrm{CD}^{+} \mathrm{T}$ cells. Nat Immunol 2013; 14: 356-363. 